\title{
Hypoxia Selectively Induces Proliferation in a Specific Subpopulation of Smooth Muscle Cells in the Bovine Neonatal Pulmonary Arterial Media
}

\author{
Julie D. Wohrley, ${ }^{*}$ Maria G. Frid, ${ }^{*}$ Elena P. Moiseeva, ${ }^{\ddagger}$ E. Christopher Orton, ${ }^{5}$ James K. Belknap, ${ }^{*}$ and Kurt R. Stenmark \\ ${ }^{*}$ Developmental Lung Biology Laboratory, University of Colorado Health Sciences Center, Denver, Colorado 80262; ${ }^{\ddagger}$ Department of \\ Biochemistry, University of Leicester, Leicester LE1 7RH, UK; and ${ }^{8}$ Colorado State University Veterinary Teaching Hospital, \\ Fort Collins, Colorado 80523
}

\begin{abstract}
Medial thickening of the pulmonary arterial wall, secondary to smooth muscle cell (SMC) hyperplasia, is commonly observed in neonatal hypoxic pulmonary hypertension. Because recent studies have demonstrated the existence of multiple phenotypically distinct SMC populations within the arterial media, we hypothesized that these SMC subpopulations would differ in their proliferative responses to hypoxic pulmonary hypertension and thus contribute in selective ways to the vascular remodeling process. Expression of meta-vinculin, a muscle-specific cytoskeletal protein, has been shown to reliably distinguish two unique SMC subpopulations within the bovine pulmonary arterial media. Therefore, to assess the proliferative responses of phenotypically distinct SMC subpopulations in the setting of neonatal pulmonary hypertension, we performed double immunofluorescence staining on pulmonary artery cryosections from control and hypertensive calves with antibodies against meta-vinculin and the proliferation-associated nuclear antigen, Ki-67. We found that, although neonatal pulmonary hypertension caused significant increases in overall cell replication, proliferation occurred almost exclusively in one, the meta-vinculin-negative SMC population, but not the other SMC population expressing meta-vinculin. We also examined fetal pulmonary arteries, where proliferative rates were high and meta-vinculin expression again reliably distinguished two SMC subpopulations. In contrast to the hypertensive neonate, we found in the fetus that the relative proliferative rates of both SMC subpopulations were equal, thus suggesting the existence of different mechanisms controlling proliferation and expression of cytoskeletal proteins in the fetus and neonate. We conclude that phenotypically distinct SMC populations in the bovine arterial media exhibit specific and selective proliferative responses to neonatal pulmonary hypertension. Distinct SMC subpopulations
\end{abstract}

J. D. Wohrley and M. G. Frid contributed equally to this work. Preliminary results were presented on May 18, 1993 at the 1993 ALA/ATS International Conference, San Francisco, California. Address correspondence to Maria G. Frid, Ph.D, Developmental Lung Biology Research, University of Colorado Health Sciences Center, 4200 East 9th Avenue, Box B131, Denver, Colorado 80262. Phone: 303-270-4498; FAX: 303-270-8353.

Received for publication 13 December 1994 and accepted in revised form 31 March 1995.

J. Clin. Invest.

(C) The American Society for Clinical Investigation, Inc.

0021-9738/95/07/0273/09 \$2.00

Volume 96, July 1995, 273-281 may, thus, contribute in unique ways to vascular homeostasis under both normal and pathologic conditions. (J. Clin. Invest. 1995. 96:273-281.) Key words: pulmonary artery • smooth muscle cells • neonatal pulmonary hypertension • proliferation - cell heterogeneity

\section{Introduction}

A significant body of evidence exists suggesting that vascular diseases such as atherosclerosis, restenosis, and neonatal pulmonary hypertension are characterized by a modulated proliferative behavior of arterial smooth muscle cells (SMCs) ${ }^{1}(1-4)$. However, it is unclear whether all SMCs respond equally to pathophysiologic stimuli or if there are specific subpopulations of SMCs which demonstrate enhanced proliferative responses and thus contribute in unique ways to the vascular remodeling process. The fact that the normal systemic and pulmonary arterial media is comprised of phenotypically diverse SMC populations (5-9) raises the possibility that not all vascular SMCs contribute equally to the proliferative changes observed in vascular disease states. Further, in vitro studies have demonstrated that multiple arterial SMC populations, which exhibit unique responses to growth promoting and inhibiting stimuli, can be isolated and maintained in culture (10-15). We thus hypothesized that, in vivo, phenotypically distinct SMC subpopulations within the bovine pulmonary arterial media would exhibit different proliferative responses to hypoxia-induced pulmonary hypertension and, thus, contribute in unique and selective ways to the medial thickening observed in hypertensive vessels.

To test this hypothesis we used as a model neonatal calves with hypoxic pulmonary hypertension, because $(a)$ neonatal calves respond to hypoxia by rapidly developing severe pulmonary hypertension and thickening of the pulmonary arterial wall $(16-19),(b)$ thickening of the pulmonary arterial media in the neonate is caused largely by cell hyperplasia $(20)$, and $(c)$ the proliferative response in the arterial media of the neonatal calf occurs primarily in the outer media (20) where two distinct SMC subpopulations can be identified on both a structural basis and the pattern of cytoskeletal protein expression (5). To distinguish SMC populations in the arterial media we chose as a marker the muscle-specific cytoskeletal protein meta-vinculin since it has been previously shown (20) that, throughout all the development, only a specific subpopulation of SMCs in the outer media expresses meta-vinculin while all other medial SMCs do not.

1. Abbreviations used in this paper: $\alpha$-SM-actin, $\alpha$-smooth muscle actin; BrdU, bromodeoxyuridine; M-VN, meta-vinculin; PA, pulmonary artery; $\mathrm{P}_{\mathrm{B}}$, barometric pressure; $\mathrm{SM}$-myosin, smooth muscle myosin; SMC, smooth muscle cell. 
To assess proliferative responses of the two phenotypically distinct SMC subpopulations, we performed indirect immunofluorescence analysis on cryosections of the main and right or left pulmonary arteries from neonatal calves using a double immunostaining technique. A monoclonal antibody Ki-67 against the proliferation-associated nuclear antigen was used to determine the proliferative state of the cell (21), while affinity purified polyclonal antibodies against meta-vinculin were used to distinguish two arterial SMC subpopulations (5). Fetal main pulmonary arteries were also examined utilizing similar methodology because the response to stress in distinct medial SMC populations of the hypertensive newborn might have reflected mechanisms held over from fetal life. In addition, because wall stresses in the main pulmonary artery of the fetus are similar to those in the hypertensive neonate, we were able to compare proliferative responses in distinct cell populations to similar stimuli at two unique developmental time points.

We found, in pulmonary arteries of neonatal calves with hypoxia-induced pulmonary hypertension, that SMC proliferation was significantly increased but that it occurred almost exclusively in a specific subpopulation of meta-vinculin-negative SMCs. In the fetus, on the other hand, where the overall rate of replication was similar to that in the hypertensive neonate, proliferation occurred nearly equally in both arterial SMC subpopulations (meta-vinculin-positive and meta-vinculin-negative). Therefore, these data suggest the existence of distinct mechanisms controlling SMC proliferation and differentiation not only in fetus and neonate but also in distinct neonatal arterial SMC subpopulations.

\section{Methods}

Animal model. Pulmonary hypertension was induced in newborn male Holstein calves by placing animals at $1 \mathrm{~d}$ of age in a hypobaric chamber with barometric pressure $\left(P_{B}\right)$ of $445 \mathrm{mmHg}$ (which is equivalent to an elevation of $4300 \mathrm{~m}$ ). This exposure has previously been reported to produce severe neonatal pulmonary hypertension with characteristic hemodynamic and structural changes (16-19). Age-matched controls were kept indoors at ambient altitude $\left(1500 \mathrm{~m}, \mathrm{P}_{\mathrm{B}}=640 \mathrm{mmHg}\right)$. Animal care procedure and hemodynamic measurements were carried out as previously described (16). Animals were sacrificed at 2, 8, and $15 \mathrm{~d}$ of age ( $n=3$ of each age, hypoxic and control) and tissue samples were isolated immediately.

To assess arterial cell proliferation/DNA synthesis, calves were injected intravenously with the thymidine analog bromodeoxyuridine (BrdU) (Sigma Chemical Co., St. Louis, MO), $10 \mathrm{mg} / \mathrm{kg}$, at 17, 9, and $1 \mathrm{~h}$ before death as previously described (20). All animal studies were performed after review and approval of both the University of Colorado and Colorado State University Animal Care and Use Committees.

Tissue samples. Freshly isolated rings ( $\sim 1 \mathrm{~cm}$ long) from the main and the right or left main pulmonary arteries were taken from 2-, 8and 15-d-old calves with hypoxia-induced pulmonary hypertension as well as from age-matched control animals ( $n=3$ in each group). Specimens of the main pulmonary artery were also obtained from fetuses of 140,160,180, and 240-265 d of gestation ( $n=3$ in each age group) (term in the bovine species is $280 \mathrm{~d}$ ). The gestation ages were calculated according to the method of Evans and Sack (22). For immunofluorescence analysis whole rings of fetal arteries were processed, whereas arterial rings from the neonatal calves were cut in half and both sectors of the artery were then processed. Arterial segments were embedded in O.C.T. Compound (Miles Inc., Kankakee, IL), frozen slowly in cold hexane to prevent tissue fracturing and stored at $-70^{\circ} \mathrm{C}$ until use. For routine histochemistry and BrdU staining arterial rings were fixed in alcohol-based fixative (Omnifix; Xenetics Biomedical, Tustin, CA) overnight, then transferred to $70 \%$ ethanol and stored at room temperature until use.

Frozen tissue samples were cut at $5 \mu \mathrm{m}$, air-dried, fixed in absolute acetone for $5 \mathrm{~min}$ at room temperature and processed for immunofluorescence staining as further described. For routine histochemistry and BrdU staining fixed tissues were dehydrated, embedded in paraffin, and sectioned at $4 \mu \mathrm{m}$ thickness.

Antibodies. The following antibodies were utilized in this study. Monoclonal anti-BrdU antibody (Becton Dickinson, Mountain View, CA) was used at a dilution of $1: 100$ in PBS containing $0.1 \%$ BSA/ $0.5 \%$ Tween-20. Biotinylated horse anti-mouse IgG and avidin/biotinhorseradish peroxidase complex (Pierce, Rockford, IL) were applied at dilutions recommended by the supplier. Monoclonal anti- $\alpha$-smooth muscle actin ( $\alpha$-SM-actin) and rabbit anti-actin antibodies (both purchased from Sigma Chemical Co.) were used at dilutions 1:100 and 1:50, respectively. Monoclonal antibody SM-M5 against smooth muscle myosin heavy-chain isoforms has been previously described (5) and was used as undiluted hybridoma culture supernatant. Rabbit antisera against desmin was purchased from Dako Corp. (Carpinteria, CA) and used at a dilution of 1:200. Monoclonal antibody against calponin has been previously described (23) and was used as undiluted hybridoma culture supernatant. Affinity-purified polyclonal anti-meta-vinculin antibodies (termed as $\mathrm{M}-\mathrm{VN}$ ) were used in this study at 1:10 dilution. These antibodies were raised in rabbits against a fusion protein containing glutathione-S-transferase and a fragment of the vinculin protein (908-916 amino acid residues) with a meta-vinculin insertion (24), and have been previously characterized (5). The monoclonal antibody $\mathrm{Ki}-67$ against nuclear-associated antigen of proliferating cells (found throughout the cell cycle $\left(G_{1}, S, G_{2}\right.$, and $M$ phases $)$ and absent in resting $\left(G_{0}\right)$ cells) was purchased from Dako Corp. and used at a dilution of $1: 25(21,25)$.

Immunostaining and routine histochemistry. Routine hematoxylin/ eosin staining was performed using standard histological techniques. To identify cells that incorporated the thymidine analog, bromodeoxyuridine (BrdU), immunoperoxidase staining was performed as described elsewhere (26).

For indirect single-label immunofluorescence staining, acetone-fixed frozen tissue sections were incubated in blocking solution (phosphate buffered saline [PBS] containing 5\% calf serum) for 5-10 min and then incubated with primary antibodies for $1 \mathrm{~h}$ at room temperature. Secondary antibodies (FITC-conjugated rabbit anti-mouse IgG or FITC-conjugated goat anti-rabbit IgG) were purchased from Calbiochem and were applied at a dilution of 1:40, each. Controls were performed in which primary antibodies were replaced by nonimmune calf serum.

For double-label immunofluorescence staining, the antibodies were applied in the following order: staining with $\mathrm{Ki}-67$ antibody was accomplished first using Biotin/Streptavidin-Texas Red detection system, then staining with polyclonal M-VN antibodies was performed using FITCconjugated anti-rabbit IgG as secondary antibodies. The stained sections were examined on Nikon Optiphot epifluorescence photomicroscope with $10 \times, 20 \times$, and $40 \times$ objectives. Colored photomicrographs were taken with Ektachrome 160T film.

Quantitative assessment of SMC proliferation. To quantitatively assess proliferation within phenotypically distinct SMC populations (meta-vinculin-positive versus meta-vinculin-negative) in the neonatal arterial media, the number of Ki-67-positive nuclei was evaluated separately for meta-vinculin-positive and meta-vinculin-negative cells. A minimum of 500 replicating ( $\mathrm{Ki}-67$-positive) cells was counted for each section throughout the entire vessel wall. Three separate counts on different sections were performed for each animal.

To determine relative proliferation within distinct SMC populations (meta-vinculin-positive versus meta-vinculin-negative) in the fetal arterial media, a triple staining procedure using monoclonal $\mathrm{Ki}-67$, polyclonal M-VN antibodies, and the nuclear stain, Hoechst-33342 (27), 

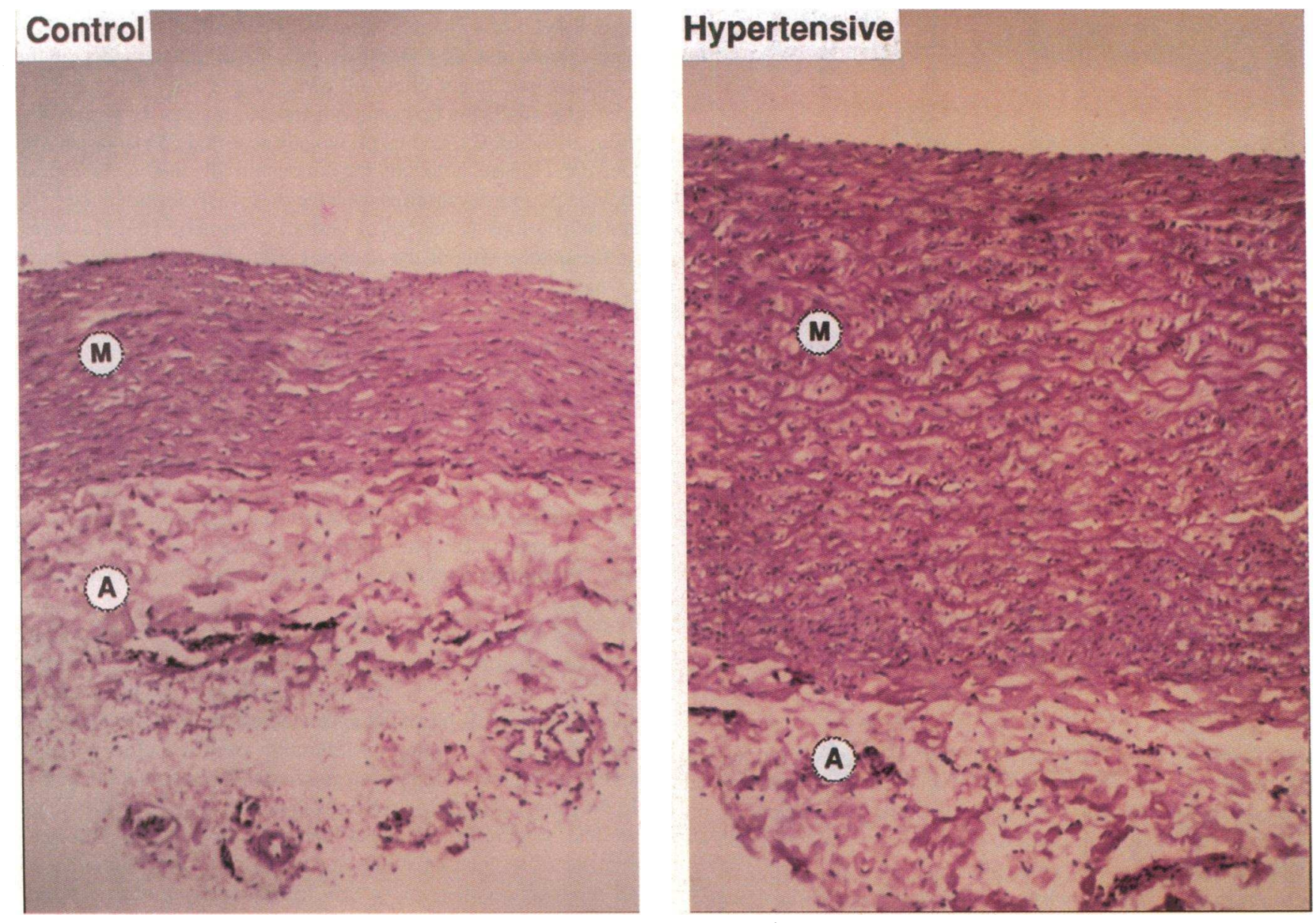

Figure 1. Medial wall thickening in neonatal pulmonary hypertension. Photomicrographs of the right pulmonary arteries from control and hypertensive 14-d-old calves (hematoxylin/eosin staining). The pulmonary arterial media $(M)$ of the calf with severe neonatal pulmonary hypertension is much thicker than that of an age-matched control animal. The adventitia $(A)$ is shown although not in its entirety in the hypertensive vessel.

was performed on 140,180, and 260-d-old fetuses as follows. Doublelabel immunostaining with $\mathrm{Ki}-67$ and $\mathrm{M}-\mathrm{VN}$ antibodies was accomplished first as described above, then $2 \mathrm{mM}$ Hoechst-33342 solution in Tris-buffer was applied for $5 \mathrm{~min}$ at room temperature. The stained sections were thoroughly washed in PBS and examined on the Olympus epifluorescence photomicroscope with filters appropriate for each stain. Black and white photomicrographs of the same field were taken for each stain separately using the appropriate filter: Quantitative analysis of proliferation within each distinct cell population (meta-vinculin-positive or meta-vinculin-negative) was accomplished in the following order. First, the total number of meta-vinculin-positive cells was evaluated by overlaying photomicrographs of Hoechst-33342 and meta-vinculin staining. Then, the number of replicating cells within a meta-vinculinpositive population was assessed by overlaying photomicrographs of Ki-67 and meta-vinculin staining. The same procedure was repeated for meta-vinculin-negative cell population. The data are presented as the percentage of replicating cells within the meta-vinculin-positive or metavinculin-negative cell population (see Fig. 9). One and two way ANOVA followed by Scheffe's multiple comparison test were utilized for statistical analysis. Data were considered significantly different if $P<0.05$.

\section{Results}

Increased pulmonary artery pressure and structural remodeling of the media in hypoxia-exposed neonatal calves. The normal decline in pulmonary artery pressure occurring after birth was inhibited by exposure of neonatal calves to hypobaric hypoxia. Pulmonary artery pressures in hypertensive and control animals were $72 \pm 16$ versus $47 \pm 8$ on day $2,126 \pm 23$ versus $31 \pm 1$ on day 8 and $118 \pm 10$ versus $25 \pm 4$ on day 15 , respectively. Thus, hypoxia caused not only an inhibition of the normal postnatal decline in pulmonary arterial pressure but also the development of severe pulmonary hypertension in neonatal calves.

Histologic examination of the main and right pulmonary arteries demonstrated dramatic medial thickening in vessels from hypertensive animals (Fig. 1). Apparent increases in the number of nuclei as well as the size of cells within the media were noted.

Increased proliferation in arterial SMCs in response to hypoxia-induced pulmonary hypertension. The contribution of cell proliferation and/or increased DNA synthesis to hypoxia-induced vessel wall thickening was addressed by examining BrdU incorporation in medial SMCs of the main pulmonary artery of hypertensive and control calves at 2,8 , and $15 \mathrm{~d}$ of age (Fig. 2 ). In the arteries of control animals the labeling index (percentage of nuclei incorporating $\mathrm{BrdU}$ versus total nuclei number) was highest on day $2(1.0 \%)$ and progressively decreased over the first 2 wk of life. In the arteries of hypertensive animals BrdU incorporation was increased on days 8 and 15, compared 


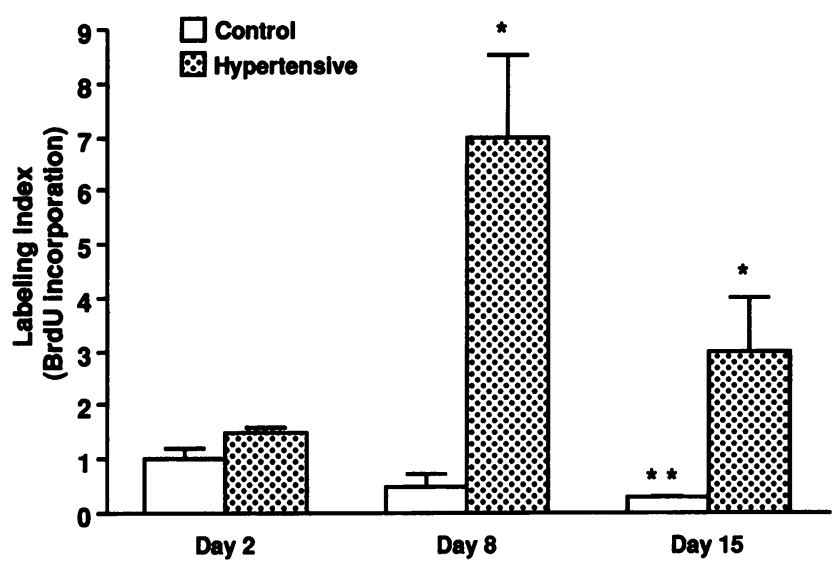

Figure 2. BrdU labeling is increased in the pulmonary arterial media of neonatal calves with hypoxic pulmonary hypertension. The BrdU labeling index (nuclei incorporating BrdU/total nuclei $\times 100$ ) in pulmonary arteries of control calves (open bars) decreased postnatally (**P $<0.05$ compared with day 2 ). In neonatal calves with pulmonary hypertension (stippled bars), on the other hand, not only was this decline prevented, but significant increases in proliferation, compared to age-matched control animals, were observed $(* P<0.05) .(n=3$ in each age group at each time point).

with age-matched controls, where it was 14 - and 10 -fold higher, respectively, than in controls. Our previous studies in this model had demonstrated that the increased BrdU labeling index reflected actual cell proliferation and not simply increased DNA synthesis (20).

Heterogeneous response of phenotypically distinct pulmonary artery SMC subpopulations to hypoxia-induced pulmonary hypertension. The possibility that not all medial SMCs participated equally in the proliferative responses observed, was raised by our recent observations demonstrating that the bovine arterial media is comprised of multiple phenotypically distinct SMC populations (5). Fig. 3 shows that the media of the neonatal bovine pulmonary artery is heterogeneous both with regard to structural organization and SMC phenotype. Structural heterogeneity was revealed by gross microscopic examination of sections stained with an antibody against total actin which reacts with all cells in the vessel wall (Fig. $3 A$ ). Compact cell clusters ( "C"' in Fig. $3 A$ ) composed of longitudinally oriented cells, were identified within the outer media. These cell clusters were interspersed among more typical appearing thin, elongated and circumferentially oriented SMCs ("i" in Fig. $3 \mathrm{~A}$ ). Diversity in SMC phenotype was demonstrated by the differential expression of the high molecular weight variant of vinculin, metavinculin, by a specific subpopulation of SMCs in the outer media. Cells in compact clusters ("C"' in Fig. 3, $A$ and $D$ ) expressed meta-vinculin, whereas other cells ("i" in Fig. 3, $A$ and $D$ ) did not. Fig. 3 shows that in the outer media of the neonatal pulmonary artery all SMCs expressed a-smooth muscle-actin ( $\alpha$-SM-actin), smooth muscle-myosin (SM-myosin) (Fig. 3, $B$ and $C$ ), and as previously reported (5) calponin and desmin. (Please, note that in the inner-middle media of neonatal bovine pulmonary arteries we have previously reported the existence of a mosaic pattern of desmin expression [5]. However, in the outer media of the neonatal pulmonary artery the pattern of desmin expression in SMCs was homogeneous.)

To determine if hypoxic pulmonary hypertension stimulated proliferation equally in all SMC populations, or if one subpopulation was preferentially effected, we used double-labeling immunofluorescence analysis. Ki-67, an antibody against a nuclear-associated proliferative marker, was used to assess SMC proliferation. Concurrently, meta-vinculin expression was evaluated to distinguish the two SMC populations within the pulmonary arterial media. Proliferation (Ki-67-positive nuclei) was seen to occur in cells throughout the entire media but rarely, if ever, within the population of SMCs expressing meta-vinculin (Fig. 4).

Quantitative analysis of proliferation within the two SMC populations (meta-vinculin-positive versus meta-vinculin-negative) in the arterial media of hypertensive neonatal calves showed that at all postnatal ages studied, in both the main and right pulmonary arteries, proliferation occurred almost exclusively in the meta-vinculin-negative SMC population (Fig. 5). In the pulmonary arteries of 8-d-old hypertensive calves (where we had observed the highest labeling index) (Fig. 2) the percentage of replicating/meta-vinculin-postive SMCs relative to all replicating cells, was $<5 \%$ in the right pulmonary artery (right PA) and $<2 \%$ in the main pulmonary artery (main PA) (Fig. 5).

Homogeneous pattern of proliferation in fetal arteries. To determine if the remarkable differences in proliferative behavior observed in the hypertensive neonate existed under other conditions associated with high rates of SMC replication, we examined the pattern of SMC proliferation in fetal pulmonary arteries, where pressure and wall stress are high (as in hypertensive neonatal animals) and where a high index of proliferation exists (28).

Two phenotypically distinct SMC subpopulations (metavinculin-positive and meta-vinculin-negative), identical to those in neonatal arterial media, were also identified in the fetal arteries (140-270 d of gestation). The proliferative behavior of these two SMC populations was analyzed by double-labeling immunofluorescence utilizing Ki-67 and M-VN antibodies. In contrast to the hypertensive neonatal arteries (see Fig. 4 and $5)$, coexpression of $\mathrm{Ki}-67$ antigen and meta-vinculin within the same cell was frequently observed in the fetal arteries of all

Figure 3. Meta-vinculin expression differentiates two smooth muscle cell subpopulations in the arterial media. Indirect immunofluorescence staining of serial cryosections of neonatal bovine pulmonary artery (only outer media is shown) with antibodies against total actin $(A), \alpha$-SM-actin ( $B$ ), SM-myosin $(C)$, and meta-vinculin $(D)$. Only one SMC subpopulation ("C'") expresses meta-vinculin, whereas other SMCs ("i') do not. Note that both SMC subpopulations express $\alpha$-SM-actin and SM-myosin.

Figure 4. Proliferation in the pulmonary arteries of hypertensive neonatal calves occurs predominantly in the meta-vinculin-negative SMC population. Double label immunofluorescence staining of cryosections from four pulmonary arteries (only outer media is shown) of hypoxic hypertensive neonatal calves. Expression of Ki-67 antigen (arrowheads; Texas Red, red) was observed only in the meta-vinculin-negative SMC subpopulation and not in meta-vinculin-positive cells (indicated by an asterisk; fluorescein, green). 

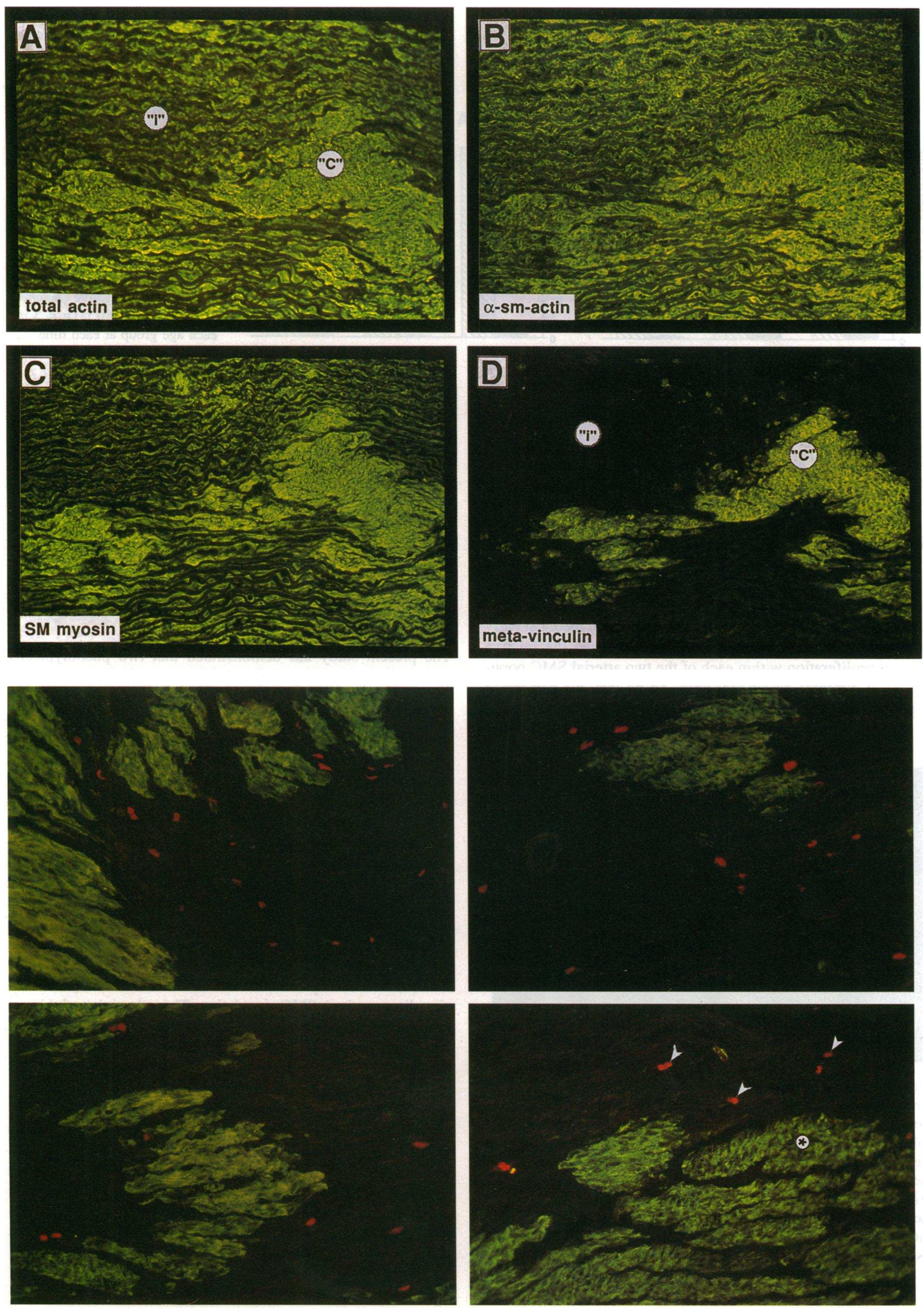
Figure 5. Quantitative analysis demonstrates that proliferation occurs almost exclusively in the meta-vinculin-negative cell population in the pulmonary arteries of hypertensive neonatal calves.

Right PA

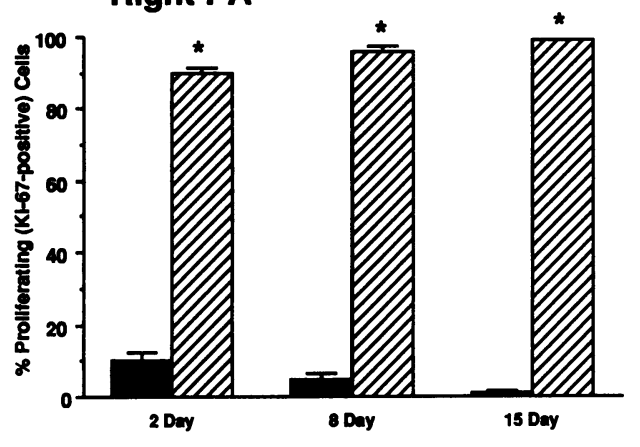

Main PA

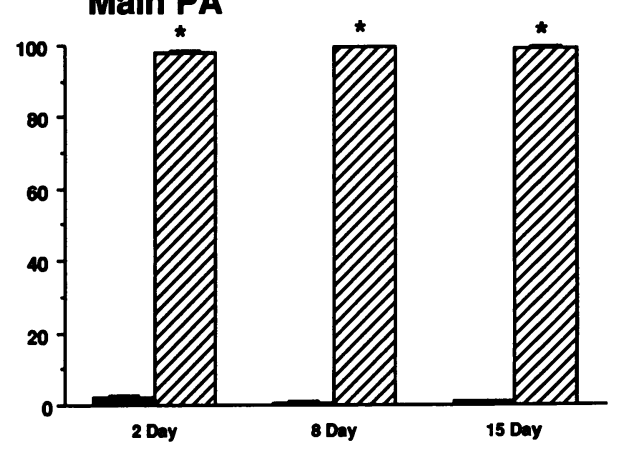

Double-label immunofluores-

cence staining of right and main pulmonary artery cryosections was utilized to quantitatively assess proliferation (as determined by a nuclear $\mathrm{Ki}-67$ antigen expression) in two phenotypically distinct SMC subpopulations, metavinculin-positive (solid bars) and meta-vinculin-negative (hatched bars $)(* P<0.001)$. ( $n=3$ in each age group at each time point). gestation ages analyzed (Fig. 6). Quantitative analysis showed that from $20 \%$ (in the 140-d-old fetuses) to $40 \%$ (in the 265 d-old fetuses) of all replicating cells simultaneously expressed meta-vinculin (Fig. 7).

Because we had previously shown that the clusters of metavinculin-positive cells increased in size and number in the arterial media as gestation progressed (5), the data shown in Fig. 7 raised the question as to whether the two SMC subpopulations in the fetal arterial media had different proliferative capabilities or if the observed differences simply reflected a lower proportion of meta-vinculin-positive cells in the arterial media at early fetal ages. Therefore, we quantitatively analyzed the relative rates of proliferation within each of the two arterial SMC populations at different gestational ages. To do this, we performed triple-labeling immunofluorescence staining utilizing Ki-67 and

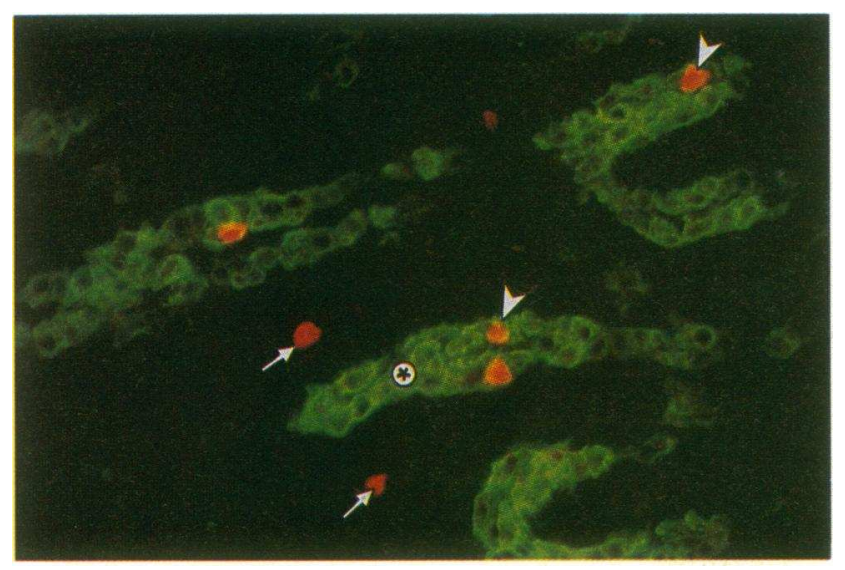

Figure 6. Ki-67 nuclear antigen and meta-vinculin are frequently coexpressed in smooth muscle cells of the fetal pulmonary arterial media. Double-label immunofluorescence staining of a 265-d-old fetal main pulmonary artery (only outer media is shown) using antibodies against the proliferation-associated nuclear antigen, Ki-67 (Texas Red, red) and the premembrane cytoskeletal protein meta-vinculin (fluorescein, green). In contrast to the neonate (see Figs. 4 and 5), in the fetus both meta-vinculin-positive and meta-vinculin-negative SMCs (arrowheads and arrows, respectively) express $\mathrm{Ki}-67$, indicating active DNA synthesis/proliferation in both cell populations.
M-VN antibodies, as well as the nuclear stain Hoechst-33342 on main pulmonary arteries of $135-145,180-185$, and $240-$ 265-d-old fetuses so that labeling indices in the two cell populations could be calculated (Fig. 8). Fig. 9 shows that the metavinculin-positive and meta-vinculin-negative SMC populations have equal proliferative rates at each gestational age. Thus, in the fetal arterial media both SMC populations had similar proliferative capabilities.

\section{Discussion}

The present study has demonstrated that two phenotypically distinct smooth muscle cell (SMC) subpopulations, coexisting at the same site within the bovine neonatal pulmonary arterial media, exhibit markedly different proliferative responses to the same pathophysiologic stimulus, i.e., hypoxic pulmonary hypertension. The existence of distinct SMC populations was confirmed by both morphological and immunobiochemical studies. Marked and consistent differences between arterial SMCs with regard to the patterns of their arrangement, orientation, and expression of meta-vinculin, a muscle-specific cytodifferentiation-related marker, were observed in every section from all large elastic pulmonary arteries examined. The observation that these two SMC populations in neonatal pulmonary arteries had remarkably different proliferative responses to hypoxia-induced pulmonary hypertension was confirmed by quantitative studies demonstrating that, in every animal, and at each postnatal age studied, $>95 \%$ of proliferation occurred within one (the metavinculin-negative) SMC population. The selectivity of this response in the neonate was further strengthened by observations demonstrating that in the fetus both SMC populations exhibited nearly equal rates of proliferation. Therefore, in the neonatal bovine pulmonary arterial media one specific SMC subpopulation responded to hypoxic pulmonary hypertension with increased proliferation, whereas another SMC subpopulation was resistant.

Our assertion that these were distinct responses exhibited by two different SMC populations and not the result of temporal and transient downregulation of meta-vinculin expression in replicating cells of a single SMC population, was further supported by the following observations: $(a)$ meta-vinculin expres- 


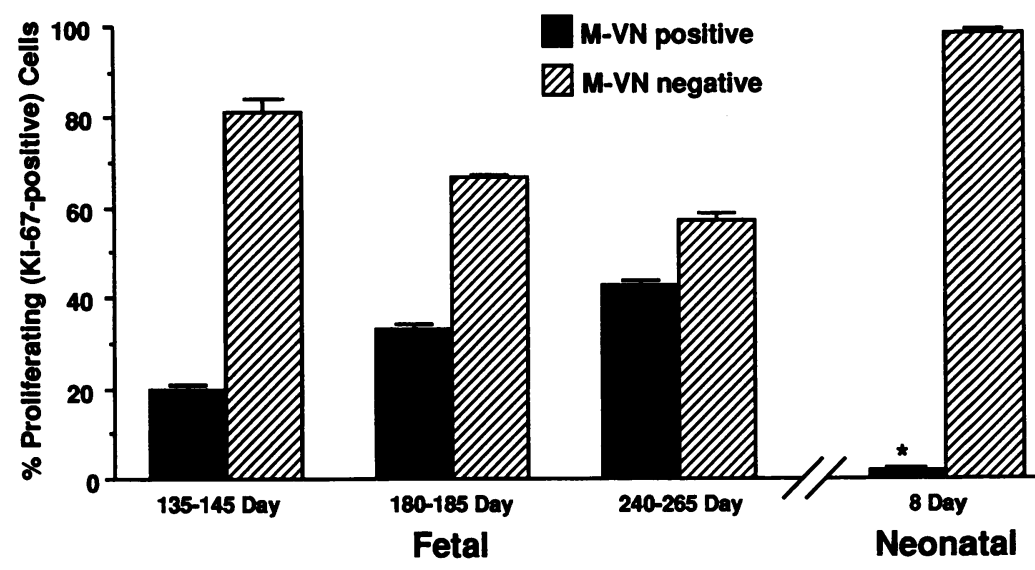

Figure 7. Quantitative analysis demonstrates that, in contrast to the hypertensive neonate, proliferation in fetal arteries occurs in both the meta-vinculin-positive and the meta-vinculin-negative SMC subpopulations. Double-label immunofluorescence staining of main pulmonary arteries of fetuses $(n=3$ at each gestational age) was used to quantitatively assess proliferation (as determined by a nuclear $\mathrm{Ki}-67$ antigen expression) in two phenotypically distinct SMC subpopulations, metavinculin-positive (solid bars) and meta-vinculin-negative (hatched bars). ( $* P<0.05$ from proliferation in the meta-vinculin-positive SMC subpopulation [solid bars] in fetal arteries at all gestational ages studied.)

sion was not found to be an exclusive prerogative of a nonproliferative SMC phenotype, since in fetal arteries, coexpression of meta-vinculin and the proliferation-associated antigen, $\mathrm{Ki}-67$, was commonly observed; and $(b)$ in the hypertensive neonate, SMCs without proliferative response to hypoxic stimulus could be identified not only on the basis of meta-vinculin expression but also by their unique arrangement pattern and longitudinal orientation. It seems unlikely that the observed differences in proliferative responses between the two SMC populations were due to stress being differently imposed on cells oriented circumferentially or longitudinally within the vessel wall because in the fetus, where the pulmonary arterial pressures and wall tension approach those in the hypertensive neonate, both SMC populations proliferate nearly equally (Fig. 9).

The hypothesis that unique SMC subpopulations comprise

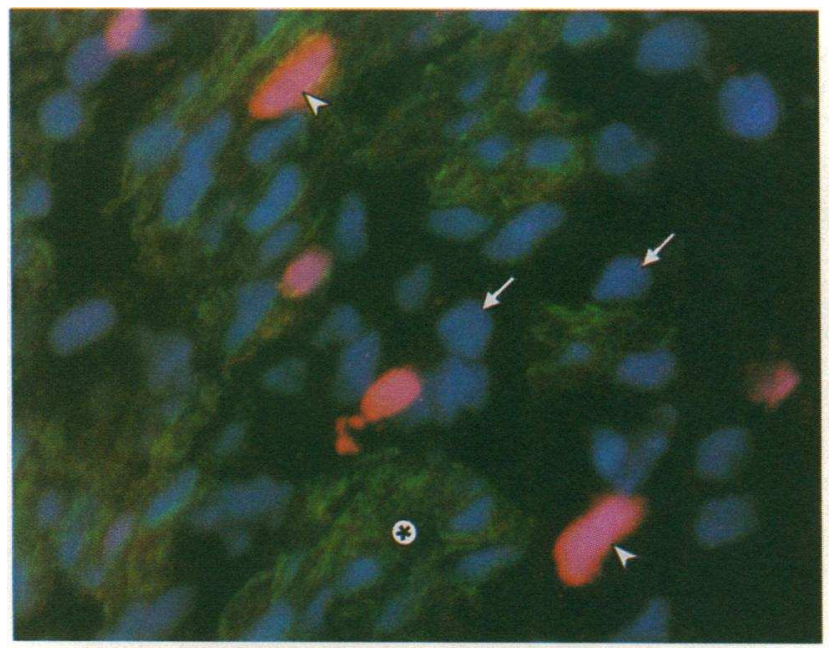

Figure 8. Triple-label immunofluorescence staining was used to determine the labeling index in two arterial cell populations. Photomicrograph of the outer media of a fetal (265-d-old) main pulmonary artery stained with antibodies against the proliferation-associated nuclear antigen Ki67 and meta-vinculin, as well as Hoechst- 33342 nuclear stain. All cell nuclei are labeled with Hoechst-33342 fluorochrome. Nuclei of nonproliferating cells appear blue (arrows). Nuclei of proliferating cells stain pink (the blue from Hoechst-33342 fluorochrome and red fluorescence of Texas Red (Ki-67) being simultaneously expressed give a pink color) (arrowheads). Cells expressing meta-vinculin are identified by their green fluorescence (indicated by an asterisk). the arterial media and respond selectively to pathologic stress has emerged from previous in vivo and in vitro studies. Several laboratories have reported the existence of SMCs of different size, shape and differentiation state in the normal arterial media $(5-8,35)$. Further, increased numbers of SMCs with an "immature" phenotype have been reported in arterial intima and/ or media in vascular diseases such as atherosclerosis, restenosis, and renovascular hypertension $(3,9,29-32)$. The presence of "less differentiated" cells in the vessel wall could be the result of either dedifferentiation or "phenotypic modulation" of differentiated medial SMCs (33) or the expansion of a preexisting subpopulation of "immature"' SMCs (34) during the pathogenesis of vascular disease. Further, although indirect, support for the concept that genetically distinct SMCs exist in the arterial media and exhibit differential proliferative responses to growth

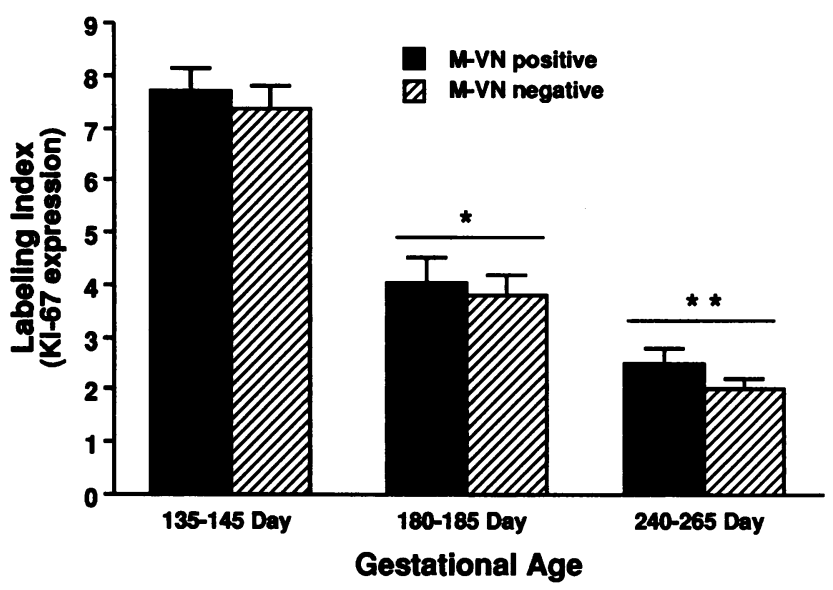

Figure 9. Labeling index for meta-vinculin-positive and -negative cell populations in the outer media is equal in the fetus. The Ki-67 labeling index (nuclei expressing $\mathrm{Ki}-67$ antigen/total number of nuclei within each SMC population [i.e., meta-vinculin-positive or meta-vinculinnegative] $\times 100$ ) was calculated for the two distinct SMC subpopulations in the outer media of early (135-140-d-old), middle (180-185d-old), and late (240-265-d-old) gestation fetal main pulmonary arteries ( $n=3$ at each gestational age). Relative rates of proliferation within both SMC subpopulations is equal at all gestational ages studied. Note that proliferation in both cell populations declines with advancing gestational age $\left({ }^{*} P<0.05\right.$ compared to 140 -d-old fetus, ${ }^{* * P}<0.05 \mathrm{com}$ pared with 180-d-old fetus). 
promoting stimuli is derived from recent in vitro studies. Several laboratories have demonstrated that at least two SMC populations, which exhibit differential responses to the same stimulus, can be isolated from normal arteries (10-15). A subpopulation of SMCs has been isolated from the normal arterial media of neonatal and adult rats and monkeys which can grow in PDGFfree media, whereas another, simultaneously isolated, SMC population requires PDGF for proliferation $(10,13)$. Other studies have shown that a subpopulation of SMCs less sensitive to the growth inhibitory effects of heparin could be selectively isolated from adult rat aortic media $(12,14)$. Two stable subtypes of SMCs were isolated from human adult carotid arteries based on markedly different growth characteristics (11). Most recently, two morphologically distinct SMC populations isolated from the mature rat aortic media were shown to differ dramatically in their ionic responses to vasoactive agonists (15). Thus, the in vivo data of the present study extend the in vivo and in vitro observations of other investigators and strongly support the idea that distinct SMC subpopulations exist in the arterial media which exhibit uniquely different responses to a given mitogenic stimulus. Specific subpopulations of medial SMCs with enhanced proliferative potential may thus contribute selectively to the process of vascular remodeling observed in neonatal pulmonary hypertension.

Significant changes in the proliferative behavior and/or potential of different SMC subpopulations appear to take place over a very short period of time between late gestation and early neonatal life. In fetal arteries, relatively high rates of proliferation were observed in all SMC subpopulations. After birth, a decline in the replication rate of both pulmonary arterial SMC populations examined was observed under normal conditions. In the setting of severe pulmonary hypertension overall SMC replication remained equal to or even greater than that observed in the late gestation fetus. However, only one subset of SMCs (meta-vinculin-negative) demonstrated a persistence of the high fetal rates of proliferation (Fig. 5). The other SMC population (meta-vinculin-positive), which had simultaneously expressed meta-vinculin and the replication-associated marker Ki-67 in the late gestation fetus (Figs. 6-9), did not proliferate under hypertensive conditions (Fig. 5). Thus it appears that dramatic changes in the mechanisms controlling cell proliferation occur at the time of birth in some but not all SMC populations. The factors regulating such dramatic changes in the proliferative potential of arterial SMCs before and after birth are not known.

In the present study we observed that in fetal arteries metavinculin, which is believed to be a cytodifferentiation-related marker, was simultaneously expressed with the proliferationassociated antigen, Ki-67. Previous studies, mostly in vitro, have suggested an inverse correlation between expression of cytodifferentiation-related cytoskeletal proteins and proliferation (36-40). Our observations in fetal arteries do not support this idea. Moreover, we observed that cells not expressing metavinculin in the outer media of fetal and neonatal arteries did express other muscle-specific contractile and cytoskeletal proteins such as $\alpha$-SM-actin, SM-myosin, calponin and desmin and coexpression of these proteins with a proliferation-associated nuclear marker Ki-67 was also routinely observed (authors' unpublished observations). These results are consistent with recent studies by other investigators demonstrating that proliferation of SMCs can occur without downregulation of muscle specific proteins traditionally associated with SMC differentiation state $(41-43)$. Thus, the traditional hypothesis regarding the existence of an inverse correlation between the expression of cytodifferentiation-associated markers and proliferation (3640) should be reconsidered. The mechanisms responsible for differences in the ability of various mitogens/growth factors to affect SMC differentiation state thus warrant further investigation.

The observation that distinct SMC subpopulations in the bovine pulmonary artery respond to a given stimulus in unique ways may have important implications not only for the human pulmonary circulation, but also for the human vascular disease states in the systemic circulation, such as atherosclerosis, restenosis and hypertension. Evidence is beginning to accumulate that the normal media of human arteries is also composed of SMCs with diverse phenotypes. If, indeed, at one site in the arterial media there exist different SMC subtypes which vary in their responses to the same pathophysiologic stimulus, the concept that such stimuli cause a single-type SMC to modulate its phenotype (33), may be incomplete. The existence of functionally heterogenic SMC populations would allow adaptive responses to pathophysiologic stimuli or injury to occur without compromising completely the homeostatic functions required of a vessel like the pulmonary artery. In the future then, different strategies will be needed to investigate the molecular mechanisms controlling proliferative behavior of distinct arterial SMC populations under both normal and pathologic conditions.

\section{Acknowledgments}

The authors wish to thank Steven Hofmeister and Miriam Bender for excellent technical assistance, and Dr. John T. Reeves for valuable discussion of the manuscript.

This work was supported by National Institutes of Health grants SCOR HL-46481, Program Project grant HL-14985, an American Lung Association Career Investigator Award to K. R. Stenmark, and NIH NRSA to J. K. Belknap.

\section{References}

1. Schwartz, S. M., G. R. Campbell, and J. H. Campbell. 1986. Replication of smooth muscle cells in vascular disease. Circ. Res. 58:427-444.

2. Schwartz, S. M., R. L. Heimark, and M. W. Majesky. 1990. Developmental mechanisms underlying pathology of arteries. Physiol. Rev. 70:1177-1209.

3. Bondjers, G., M. A. Glukhova, G. K. Hansson, Y. V. Postnov, M. A. Reidy, and S. M. Schwartz. 1991. Hypertension and atherosclerosis. Cause and effect, or two effects with one unknown cause? Circulation. 84 [suppl VI]:VI-2-VI-16.

4. Hadrava, V., J. Tremblay, and P. Hamet: 1989. Abnormalities in growth characteristics of aortic smooth muscle cells in spontaneously hypertensive rats. Hypertension. 13:589-597.

5. Frid, M. G., E. P. Moiseeva, and K. R. Stenmark. 1994. Multiple phenotypically distinct smooth muscle cell populations exist in the adult and developing bovine pulmonary arterial media in vivo. Circ. Res. 75:669-681.

6. Larson, D. M., K. Fugiwara, R. W. Alexander, and M. A. Gimbrone, Jr. 1984. Heterogeneity of myosin antigenic expression in vascular smooth muscle in vivo. Lab. Invest. 50:401-407.

7. Osborn, M., J. Caselitz, and K. Weber. 1981. Heterogeneity of intermediate filament expression in vascular smooth muscle: a gradient in desmin positive cells from the rat aortic arch to the level of the arteria iliaca communis. Differentiation. 20:196-202.

8. Zanellato, A. M. C., A. C. Borrione, L. Giuriato, M. Tonello, G. Scannapieco, P. Pauletto, and S. Sartore. 1990. Myosin isoforms and cell heterogeneity in vascular smooth muscle in developing and adult bovine aorta. Dev. Biol. 141:431-446.

9. Pauletto, P., S. Sartore, L. Giuriato, M. Scatena, D. Guidolin, G. Scannapieco, and A. C. Pessina. 1991. Computer-driven assessment of "immature"- 
type smooth muscle cells in rabbit aorta. J. Hypertension. 9(suppl 6):S180S181.

10. Schwartz, S. M., L. Foy, D. F. Bowen-Pope, and R. Ross. 1990. Derivation and properties of platelet-derived growth factor-independent rat smooth muscle cells. Am. J. Pathol. 136:1417-1428.

11. Fujita, H., K. Shimokado, C. Yutani, S. Takaichi, J. Masuda, and J. Ogata. 1993. Human neonatal and adult vascular smooth muscle cells in culture. Exp. Mol. Pathol. 58:25-39.

12. San Antonio, J. D., M. J. Karnovsky, M. E. Ottlinger, R. Schillig, and L. A. Pucak. 1993. Isolation of heparin-insensitive aortic smooth muscle cells. Growth and differentiation. Arterioscler. Thromb. 13:748-757.

13. Lemire, J. M., C. W. Covin, S. White, C. M. Giachelli, and S. M. Schwartz. 1994. Characterization of cloned aortic smooth muscle cells from young rats. Amer. J. Pathol. 144:1068-1081.

14. Barzu, T., J.-M. Herbert, A. Desmouliere, P. Carayon, and M. Pascal. 1994. Characterization of rat aortic smooth muscle cells resistant to the antiproliferative activity of heparin following long-term heparin treatment. J. Cell. Physiol. 160:239-248.

15. Neylon, C. B., P. V. Avdonin, R. J. Dilley, M. A. Larsen, V. A. Tkachuk, and A. Bobik. 1994. Different electrical responses to vasoactive agonists in morphologically distinct smooth muscle cell types. Circ. Res. 75:733-741.

16. Stenmark, K. R., J. Fasules, D. M. Hyde, N. F. Voelkel, A. Tucker, H. Wilson, and J. T. Reeves. 1987. Severe pulmonary hypertension and arterial adventitial changes in newborn calves at $4300 \mathrm{~m}$. J. Appl. Physiol. 62:821-830.

17. Stenmark, K. R., A. A. Aldashev, E. C. Orton, A. G. Durmowicz, D. B. Badesch, W. C. Parks, R. P. Mecham, N. F. Voelkel, and J. T. Reeves. 1991. Cellular adaptation during chronic neonatal hypoxic pulmonary hypertension. Am. J. Physiol. Suppl. (Oct) 261:97-104.

18. Durmowicz, A. G., E. C. Orton, and K. R. Stenmark. 1993. Progressive loss of vasodilator responsive component of pulmonary hypertension in neonatal calves exposed to 4,570 m. Heart Circ. Physiol. 34:H2175-H2183.

19. Stenmark, K. R., A. G. Durmowicz, J. D. Roby, R. P. Mecham, and W. C. Parks. 1994. Persistence of the fetal pattern of tropoelastin gene expression in severe neonatal bovine pulmonary hypertension. J. Clin. Invest. 93:1234-1242.

20. Orton, E. C., S. M. LaRue, B. Ensley, and K. R. Stenmark. 1992. Bromodeoxyuridine labeling and DNA content of pulmonary arterial medial cells from hypoxia-exposed and nonexposed healthy calves. Am. J. Vet. Res. 53:1925-1931.

21. Coltrera, M. D., and A. M. Gown. 1991. PCNA/ Cyclin expression and BrdU uptake define different subpopulations in different cell lines. J. Histochem. Cytochem. 39:23-30.

22. Evans, H. E., and W. O. Sack. 1973. Prenatal development of domestic and laboratory mammals: Growth curves, external features and selected references. Anat. Histo. Embryol. 2:11-45.

23. Frid, M. G., B. V. Shekhonin, V. E. Koteliansky, and M. A. Glukhova. 1992. Phenotypic changes of human smooth muscle cells during development: late expression of heavy caldesmon and calponin. Dev. Biol. 153:185-193.

24. Koteliansky, V. E., E. P. Ogryzko, N. I. Zhidkova, P. A. Weller, D. R. Critchley, K. Vancompernolle, P. Strasser, M. Way, M. Gimona, and V. J. Small. 1992. An additional exon in the human vinculin gene specifically encodes metavinculin-specific difference peptide. Eur. J. Biochem. 204:767-772.

25. Brown, D. C., and K. C. Gatter. 1990. Monoclonal antibody Ki-67: its use in histopathology. Histopathology. 17:489-503.

26. Sugihara, H., T. Hattori, and M. Fukuda. 1986. Immunohistochemical detection of bromodeoxyuridine in formalin-fixed tissues. Histochemistry. 85:193-195.

27. Cesarone, C. F., C. Bolognesi, and L. Santi. 1979. Improved microfluoro- metric DNA determination in biological material using 33258 Hoechst. Anal. Biochem. 100:188-197.

28. Cook, C. L., M. C. M. Weiser, P. E. Schwartz, C. L. Jones, and R. A. Majack. 1994. Developmentally timed expression of an embryonic growth phenotype in vascular smooth muscle cells. Circ. Res. 74:189-196.

29. Glukhova, M. A., A. E. Kabakov, M. G. Frid, O. I. Ornatsky, A. M. Belkin, D. M. Mukhin, A. N. Orekhov, V. E. Koteliansky, and V. N. Smirnov. 1988. Modulation of human aortic smooth muscle-specific variants of vinculin caldemon and actin expression. Proc. Natl. Acad. Sci. USA. 85:9542-9546.

30. Mosse, P. R. L., G. R. Campbell, Z. L. Wang, and J. H. Campbell. 1985. Smooth muscle phenotypic expression in human carotid arteries; I: comparison of cells from diffuse intimal thickenings adjacent to atheromatous plaques with those of the media. Lab. Invest. 53:556-562.

31. Okamoto, E., K. Imataka, J. Fuji, M. Kuro-o, K. Nakahara, H. Nishimura, Y. Yazaki, and R. Nagai. 1992. Heterogeneity in smooth muscle cell population, accumulating in the neointimas and the media of poststenotic dilatation of rabbit carotid artery. Biochem. Biophys. Res. Commun. 185:459-464.

32. Pauletto, P., A. Chiavegato, L. Giuriato, M. Scatena, E. Faggin, A. Grisenti R. Sarzani, M. Paci, P. D. Fulgeri, A. Rappelli, A. Pessina, and S. Sartore. 1994 Hyperplastic growth of aortic smooth muscle cells in renovascular hypertensive rabbits is characterized by the expansion of an immature cell phenotype. Circ Res. 74:774-788.

33. Chamley-Campbell, J. H., and G. R. Campbell. 1981. What controls smooth muscle phenotype? Atherosclerosis. 40:347-357.

34. Schwartz, S. M., M. A. Reidy, and A. W. Clowes. 1985. Kinetics of atherosclerosis: a stem cell model. Ann. NY Acad. Sci. 454:292-304.

35. Skalli, O., P. Ropraz, A. Trzeciak, G. Benzonana, D. Gillessen, and G. Gabbiani. 1986. A monoclonal antibody against a-smooth muscle actin: a new probe for smooth muscle differentiation. J. Cell Biol. 103:2787-2796.

36. Owens, G. K., A. Loeb, D. Gordon, and M. M. Thompson. 1986. Expression of smooth muscle-specific a-isoactin in cultured vascular smooth muscle cells: relationship between growth and cytodifferentiation. J. Cell. Biol. 102:343352.

37. Campbell, J. H., O. Kocher, O. Skalli, G. Gabbiani, and G. R. Campbell. 1989. Cytodifferentiation and expression of $\alpha$-smooth muscle actin mRNA and protein during primary culture of aortic smooth muscle cells. Arteriosclerosis. 9:633-643.

38. Fager, G., G. K. Hansson, A. M. Gown, D. M. Larson, O. Skalli, and G. Bondjers. 1989. Human arterial smooth muscle cells in culture: inverse relationship between proliferation and expression of contractile proteins. In Vitro Cell. \& Dev. Biol. 25:511-520.

39. Thyberg, J., U. Hedin, M. Sjolund, L. Palmberg, and B. A. Bottger. 1990. Regulation of differentiated properties and proliferation of arterial smooth muscle cells. Arteriosclerosis. 10:966-990.

40. Shanahan, C. M., P. L. Weissberg, J. C. Metcalfe. 1993. Isolation of gene markers of differentiated and proliferating vascular smooth muscle cells. Circ. Res. 73:193-204.

41. Panettieri, R. A., P. A. Yadvish, A. M. Kelly, N. A. Rubinstein, and M. I. Kotlikoff. 1990. Histamine stimulates proliferation of airway smooth muscle and induces c-fos expression. Am. J. Physiol. 259 (Lung Cell. Mol. Physiol. 3):L365L371.

42. Holycross, B. J., R. S. Blank, M. M. Thompson, M. J. Peach, and G. K. Owens. 1992. Platelet-derived growth factor-BB-induced suppression of smooth muscle cell differentiation. Circ. Res. 71:1525-1532.

43. Johnson, R. J., H. Iida, C. E. Alpers, M. W. Majesky, S. M. Schwartz, P. Pritzi, D. Gordon, and A. M. Gown. 1991. Expression of smooth muscle cell phenotype by rat mesanglial cells in immune complex nephritis. Alpha-smooth muscle actin is a marker of mesanglial cell proliferation. J. Clin. Invest. $87(3): 847-858$. 